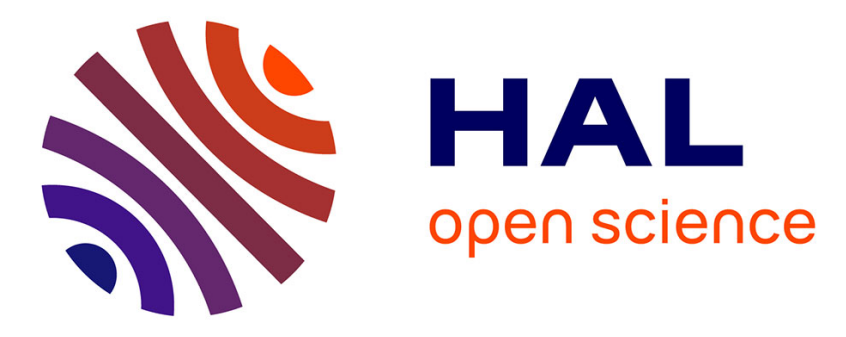

\title{
Map Matching and Lanes Number Estimation with Openstreetmap
}

Abderrahim Kasmi, Dieumet Denis, Romuald Aufrère, Roland Chapuis

\section{To cite this version:}

Abderrahim Kasmi, Dieumet Denis, Romuald Aufrère, Roland Chapuis. Map Matching and Lanes Number Estimation with Openstreetmap. 2018 21st International Conference on Intelligent Transportation Systems (ITSC), Nov 2018, Maui, France. pp.2659-2664, 10.1109/ITSC.2018.8569840 . hal-03049723

\section{HAL Id: hal-03049723 \\ https://hal.uca.fr/hal-03049723}

Submitted on 10 Dec 2020

HAL is a multi-disciplinary open access archive for the deposit and dissemination of scientific research documents, whether they are published or not. The documents may come from teaching and research institutions in France or abroad, or from public or private research centers.
L'archive ouverte pluridisciplinaire HAL, est destinée au dépôt et à la diffusion de documents scientifiques de niveau recherche, publiés ou non, émanant des établissements d'enseignement et de recherche français ou étrangers, des laboratoires publics ou privés.

\section{(c)(1)}

Distributed under a Creative Commons Attribution| 4.0 International License 


\title{
Map matching and lanes number estimation with OpenStreetMap
}

\author{
Abderrahim KASMI ${ }^{1,2}$, Dieumet DENIS ${ }^{1}$, Romuald AUFRERE ${ }^{2}$, Roland CHAPUIS ${ }^{2}$
}

\begin{abstract}
Road information, like lanes number, play an important role for intelligent vehicles (IV). Traditionally such road information are obtained through a vision-based measurement or by using a digital detailed map. In this paper, we present a new method for estimating the number of lanes using a low precision GPS receiver and OpenSteetMap (OSM). The method includes the integration of the GPS traces and OSM for a map matching. To this end we developed a probabilistic multicriteria algorithm for map matching that takes into account the accuracy of the GPS data and the attribute information of the road from OSM. Afterward we estimate the number of lanes from OSM. We tested our algorithm on a set of GPS data collected in an urban area near Paris for a total distance of $50 \mathrm{~km}$ and the overall estimation accuracy reached $83.64 \%$
\end{abstract}

\section{INTRODUCTION}

In recent years, significant progress have been made in Driver Assistance Systems (DAS) towards automated driving. Many applications, e.g., lane change assistance systems, have been the subject of several research projects [1]. For these applications knowing the number of lanes is crucial [2]. Therefore several methods have been proposed to identify the lanes. In [3], a vision-based application is proposed to estimate lanes number in road. The width of the road is evaluated using an inverse perspective mapping (IPM), then a Hough transform is applied to detect the lanes. In order to identify the lanes, the Vanishing Points estimation (VPs) based on Bayesian posterior probability are used in [4]. A combination of a Statistical Hough Transform (SHT) [5] with a Particle Filter (PF) is presented in [6] for lanes detection and lanes tracking. These vision-based applications show interesting results. However, situations like occlusions, strongly differing illuminations, and unmarked or partly marked lanes remain unresolved issues. Hence to override these limitations, other researches work on using a precise digital map to enhance the camera based systems for lanes detection [7]. However one of the main drawback of these accurate digital maps is the exorbitant cost to build them.

To overcome this constraint, researches have been made on less-expensive solutions to estimate the number of lanes. A number of researchers use GPS data to create a digital map in order to extract road information such as road curvature and lane number from it. In [8] an estimation of the number of lanes was performed by collecting the GPS traces on a road in order to calculate the Distribution Variance-Road Width

\footnotetext{
*This work has been sponsored by Sherpa Engineering and ANRT (Conventions Industrielles de Formation par la Recherche).

${ }^{1}$ Sherpa Engineering, R\&D Department, La Garenne Colombes, France. [a.kasmi, d.denis] asherpa-eng.com

2 University of Clermont Auvergne, CNRS, SIGMA Clermont, Institut Pascal, F-63000 Clermont-Ferrand, France. FirstName. Lastnameduce.fr
}

Discrete Model (DV-RWDM) [9]. These methods depend highly on the number of traces and only an accuracy of $60 \%$ was achieved [8].

On the other hand, other researches work on using the collaborative OSM mapping project for intelligent vehicles. The OSM geodata includes rich information about road. In addition to the spatial information, the geodata contains detailed information such as the name, the limitation of speed and the number of lanes [10]. As it is a collaborative project by volunteers, the accuracy of the geospatial data is undefined. In litterature, the use of the OpeenStreetMap geodata is limited to navigation tasks such a localisation [11]. In order to determine the location of the vehicle on a OSM 'link' a Map-matching algorithm is performed. The Map matching integrates positioning data with spatial road network to identify the correct 'link' on which the vehicle is travelling. In [12] a survey on the map matching methods was presented, the author classified the map matching algorithms depending on the analysis procedure as follows:

- Geometric analysis: The most commonly used mapmatching algorithm is a simple search algorithm. In this approach the closest 'node' or 'shape point' is matched to the GPS position.

- Topological analysis: In this map matching algorithm, the geometry as well as the connectivity of the links are used. In [13] a map matching based on a weighting system is proposed. Using the sensors measurement about the vehcile position, vehicle speed, yaw angle, a weighting score is calculated to choose the best link.

- Probabilistic analysis: This approach requires the definition of an elliptical confidence region around the position obtained from the GPS receiver [14] the error region is then superimposed to the road network to identify the correct link.

Other mathematical tools has been used to perfom the map matching, in [15] a probabilistic criteria is calculated to select the right link on which the vehicle travels. A Bayesian Belief Network (BBN) is used, the network takes several inputs like: the distance between the link and the vehicle position, the difference angle, the accuracy of the GPS receiver, the connectivity of the link and the direction of the link.

Compared with other related works, this paper presents a new method for map matching and lane numbers estimation exploiting OpenStreetMap. The map matching algorithm proposed in this paper is a multicriteria algorithm that takes into account several factors: geometrical, topological and probabilistic. The three methods for computing the multicriteria depend on the availability of sensors on the vehicle. 
For the number of lanes estimation, in contrast to [9], our method does not require the collection of several GPS traces. In addition, our algorithm was performed on a set of 6596 GPS points collected in an urban area for a total distance of $50 \mathrm{~km}$. An accuracy rate of $82.95 \%$ on lanes number estimation was reached.

The remainder of the paper is organized as follows: Section II introduces the data model of the OSM geodata. Section III describes the Map matching algorithm used. Section IV presents the real-world experimental results. The paper will be summarized and concluded in Section V.

\section{THE OSM GEODATA}

For local sections of the planet, an XML file containing the latest revision of the OSM map can be downloaded from the official website of OpenStreetMap. Furthermore, regularly updates of the geodata are available at that website. According to the OSM specifications, the OSM data model consists of three basic geometric elements called Nodes, Ways and Relations.

\section{A. Nodes}

Nodes $n_{i}$ are point-shaped geometric elements which are used to represent GPS points in term of latitude (lat) and longitude (lon). Moreover, Nodes are the basic points to represent the geometry of the Way.

\section{B. Ways}

Ways are used to model line-shaped geometric objects like roads, railways, pedestrianized road...etc. Throughout this paper, a single Way is defined as follows:

$$
W=\left(i d_{w}, N_{w}, T_{w}\right)
$$

Where $i d_{w}$ denotes a unique identification number of the Way. In addition, the set $N_{w}$ regroup the $m$ Nodes $n_{i}$ representing the geometry of the Way as follows:

$$
N_{w}=\left\{n_{1}, n_{2}, \ldots, n_{m}\right\}
$$

To specify the semantic of each Way, a subset $T_{w}$ of $n$ tags is related to the Way. According to the OSM specifications, each Way can have up to 255 tags. These tags are defined as follows:

$$
T_{n}=\left\{t_{1}, t_{2}, \ldots, t_{n}\right\}
$$

where $t_{i}$ indicates a single tag. Each tag consists of two elements, a key $k$ and the corresponding value $v$ :

$$
t_{i}=(k, v)
$$

As illustrated on Figure 1 an example of an OSM Way with its corresponding tags.

\section{Relations}

The element relation describes the relationship between Nodes as well as Ways. This element is not used in this paper. For more information, refer to [16].

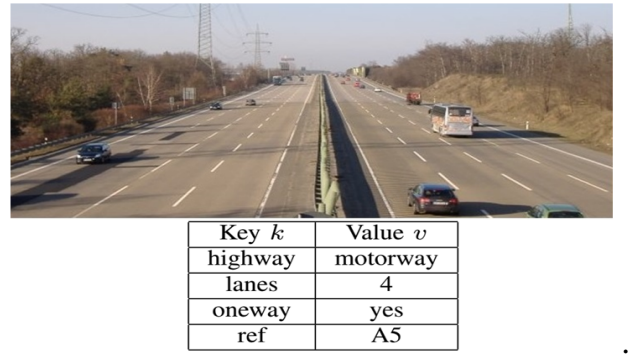

Fig. 1: Example of an OSM Way with its corresponding tags, keys and values.

TABLE I: Used OSM tags

\begin{tabular}{lcc}
\hline Description & Key & Value \\
\hline road speed limit in $\mathrm{km} / \mathrm{h}$ & maxspeed & number \\
total number of physical lanes of the Way & lanes & number \\
\hline
\end{tabular}

\section{The proceeding of the OSM data}

For this paper, only two OSM tags from the 255 possible are used. The tags used are listed in the Table I.

The OSM map consists of a set of Ways. To increase the robustness of our map matching a preprocessing stage is performed. Only Ways representing roads on which the vehicle may travel are selected as shown on Figure 2.

\section{MAP MATCHING ALGORITHM}

As discussed before, an OSM map consists of a set of Ways, each Way is constructed from multiple Nodes. In our work we represent each Way by a set of segments. In other words, belonging to segment is equivalent to belonging to an OSM Way. So the map matching task can be reformulated as matching a GPS point with a segment. In the remaining sections, words segment and Way are switchable.

To identify the Way matched with a GPS point, it is necessary to perform for each GPS node a 'discrimination stage' to remove all the Ways which are not suitable for the map matching. Afterwards, an OSM Way is chosen from the remaining OSM Way candidates by calculating a probabilistic criterion. In our paper, three methods for calculating

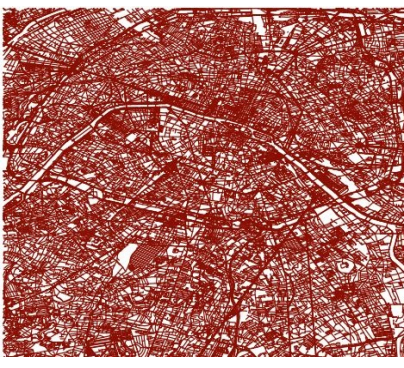

(a) Before

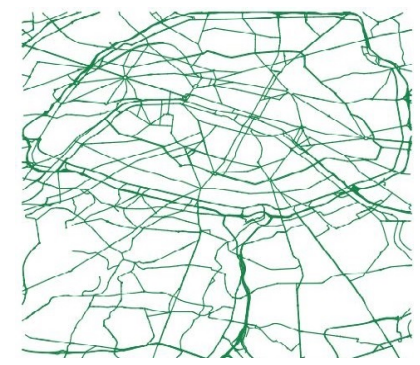

(b) After
Fig. 2: OSM map before preprocessing stage Fig (a) and after preprocessing stage Fig (b) 
this criterion are presented. Finally a Way with unique identification number is selected. Using this identification number, the number of lanes is extracted from the OSM geodata. A general presentation of the algorithm is shown in Figure 3.

In the following discussions, we will first illustrate the main factors we adopted for the discrimination stage. Thereafter we present the probabilistic criterion adopted to the map matching.

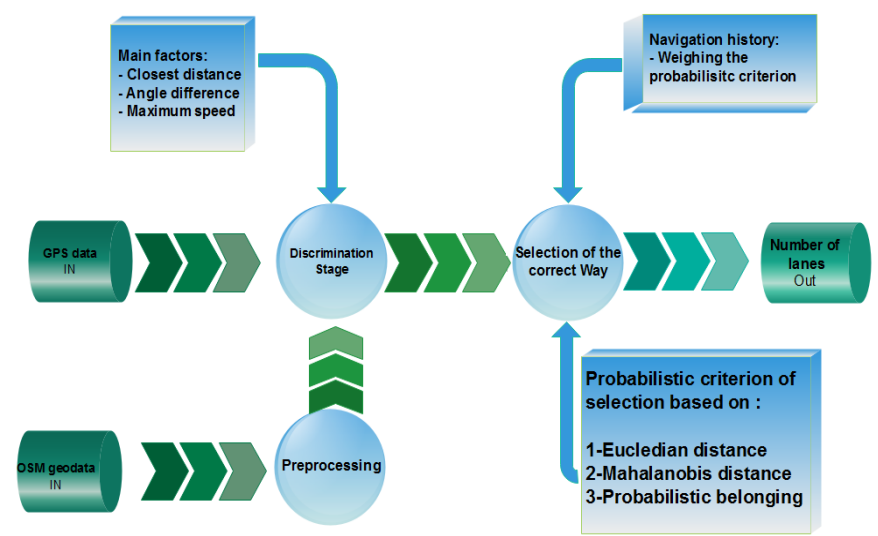

Fig. 3: Presentation of the algorithm

\section{A. Discrimination stage}

The aim of this stage is to remove all the Ways which are not suitable for map matching depending on several factors:

1) Distance to road: The distance between two geographical objects can be represented by three types of distances. As illustrated in Figure 4, this distance can be a perpendicular distance $\left(d_{0}\right)$ or a distance from point to an end point $\left(d_{1}\right.$ and $d_{2}$ ).

In our work, we take the closest distance as being the shortest distance to a Way ( $d_{0}$ in Figure $4 \mathrm{a}$ and $d_{2}$ in Figure $4 \mathrm{~b}$ ).
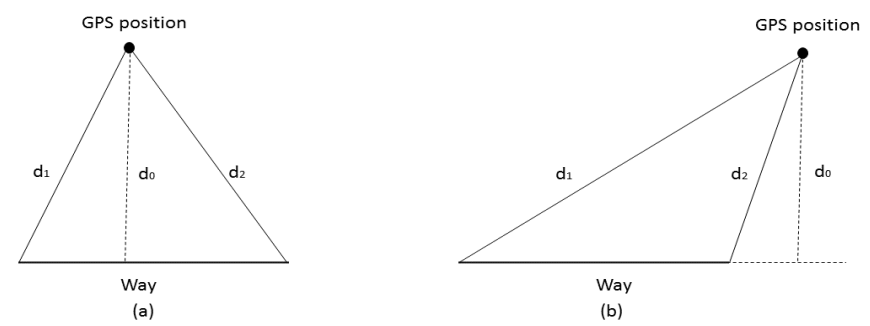

Fig. 4: Closest distance between a GPS position and a Way $\left(d_{0}\right.$ in (a) and $d_{2}$ in (b))

Ways with a distance smaller than a threshold are picked as Way candidates.

2) Angle difference: As shown in Figure 5, we compute the angle difference between the GPS trace and the Way. We retain the Ways that have an angle difference less than $90^{\circ}$.

3) Speed difference: In addition to lon, lat information, the GPS receiver provides information about the speed of the vehicle, we compare this speed to the value of the tag

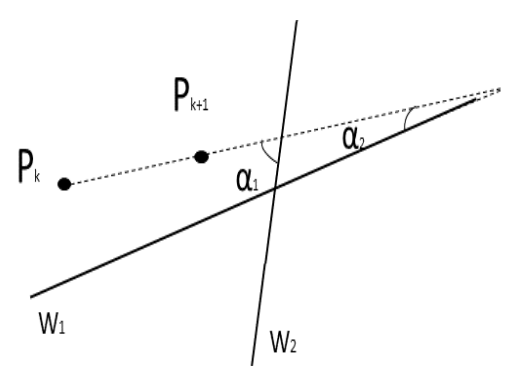

Fig. 5: Difference of angles $\alpha_{1}, \alpha_{2}$ between GPS trace and Ways $W_{1}, W_{2}$

"maxspeed" for every Way candidates. If the speed of the vehicle is greater than a threshold then the Way is eliminated. The threshold is defined as the sum of the limitation speed, from the tag "maxspeed", plus $40 \mathrm{~km} / \mathrm{h}$.

\section{B. The probabilistic criteria}

The distance and orientation criteria are not always sufficient to select the right Way. There might still be an ambiguity on choosing the right Way, as pictured on Figure 6.
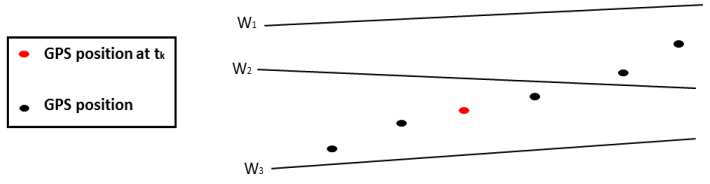

Fig. 6: Ambiguity to select the correct Way at $t_{k}$ between $W_{1}, W_{2}$ and $W_{3}$

In order to choose the right Way, we define a probabilistic selection criterion allowing to take into account the uncertainty on the choice of the Way on which the vehicle travels. In this paper, three methods of calculating this probabilistic criterion are presented:

- Probabilistic criterion based on Euclidean distance,

- Probabilistic criterion based on Mahalanobis distance,

- Probabilistic criterion based on the probability of belonging to a segment.

1) Probabilistic criterion based on Euclidean distance: The map matching task can be formulated as the calculation of the highest posterior probability of a GPS measurement $Z_{k}$ belonging to a Way $W_{i}$ :

$$
\underset{i}{\arg \max } P\left(W_{i} \mid Z_{k}\right)
$$

By assuming the uniformly distributed prior probability for the Ways $P\left(W_{i}\right)$ and using Bayes formula [17], we get:

$$
\underset{i}{\arg \max } p\left(W_{i} \mid Z_{k}\right)=\underset{i}{\arg \max }\left(\frac{p\left(Z_{k} \mid W_{i}\right) P\left(W_{i}\right)}{p\left(Z_{k}\right)}\right)
$$

Since $\mathrm{p}\left(Z_{k}\right)$ is constant for each Way candidate, then we have:

$$
\underset{i}{\arg \max } p\left(W_{i} \mid Z_{k}\right) \equiv \underset{i}{\arg \max }\left(p\left(Z_{k} \mid W_{i}\right) P\left(W_{i}\right)\right)
$$


Since $\mathrm{p}\left(W_{i}\right)$ is uniformly distributed, we get:

$$
\underset{i}{\arg \max } P\left(W_{i} \mid Z_{k}\right) \equiv \underset{i}{\arg \max } p\left(Z_{k} \mid W_{i}\right)
$$

The probability $p\left(Z_{k} \mid W_{i}\right)$ is modeled by two random independent variables :

- The distance to a road $d$ is modeled as zero mean, normally distributed random variable with standard deviation $\sigma_{d}$ described as follows:

$$
\sigma_{d}=\epsilon \cdot D O P^{1}
$$

Due to the uncertainty of the GPS data and the OSM map (beetween 6-9 $m$ [16]). The theoretical error in distance $\epsilon$ was chosen to be $15 \mathrm{~m}$.

- The angle difference $\theta$ is modeled as zero mean, normally distributed random variable with standard deviation $\sigma_{\theta}$ defined as being inversely proportional to the speed of the vehicle $v$ :

$$
\sigma_{\theta} \propto \frac{1}{v}
$$

We rewrite (8) as follows:

$$
\begin{aligned}
\underset{i}{\arg \max } P\left(W_{i} \mid Z_{k}\right) & =\underset{i}{\arg \max }\left(p_{d}\left(d\left(Z_{k}, W_{i}\right)\right) \times p_{\theta}\left(\theta\left(Z_{k}, W_{i}\right)\right)\right. \\
& =\underset{i}{\arg \max }\left(\frac{1}{\sqrt{2 \pi} \times \sigma_{d}} \cdot \exp \left(-\frac{d^{2}\left(Z_{k}, W_{i}\right)}{2 \sigma_{d}^{2}}\right) .\right. \\
& \left.\frac{1}{\sqrt{2 \pi} \times \sigma_{\theta}} \cdot \exp \left(-\frac{\theta^{2}\left(Z_{k}, W_{i}\right)}{2 \sigma_{\theta}^{2}}\right)\right) \\
& =\underset{i}{\arg \min }\left(\frac{d^{2}\left(Z_{k}, W_{i}\right)}{\sigma_{d}^{2}}+\frac{\theta^{2}\left(Z_{k}, W_{i}\right)}{\sigma_{\theta}^{2}}\right)
\end{aligned}
$$

Finally, the probabilistic criterion $C_{d}$ is calculated for every way candidate, the Way having the smallest criterion is chosen among the Way candidates:

$$
C_{d}=\left(\frac{d^{2}\left(Z_{k}, W_{i}\right)}{\sigma_{d}^{2}}+\frac{\theta^{2}\left(Z_{k}, W_{i}\right)}{\sigma_{\theta}^{2}}\right)
$$

This selection criterion does not take into account the correlation between the GPS measurements in longitudinal and lateral directions. In addition, the orientation angle of the vehicle is calculated from the GPS trace. To address these issues, an extended Kalman-filter (EKF) is used in order to get the uncertainty matrix related to the pose of the vehicle.

2) Probabilistic criterion based on Mahalanobis distance: Using the covariance matrix associated with the pose of the vehicle $\Sigma_{X}$, we compute the Mahalanobis distance for each Way candidate. To this end, we calculate the orthogonal projections of the vehicle position onto every Way candidate. Afterward, the Mahalanobis distance $C_{m}$ is calculated as follows:

$$
C_{m}=\sqrt{(\bar{X}-Y)^{T} \Sigma_{X}^{-1}(\bar{X}-Y)}
$$

- $X=(x, y, \theta)^{T}$ the current state vector representing the pose of the vehicle and $\bar{X}$ its estimation and $\Sigma_{X}$ the corresponding covariance matrix,

- $Y=\left(x_{w}, y_{w}, \psi\right)^{T}$ the vector representing the orthogonal projection on the Way.

${ }^{1}$ Dilution of precision
3) Probabilistic criterion based on the probability of belonging to a segment: To compute this probabilistic criterion, we look for the probability of a segment $[A B]$ defined by $A=\left(x_{A}, y_{A}\right)^{T}$ and $B=\left(x_{B}, y_{B}\right)^{T}$ to belong to an expected area of presence for the vehicle.

To this end, each segment $[A B]$ is modeled as a random vector $S_{A B}$ centered on $\bar{S}_{A B}$ and defined by its covariance matrix $\Sigma_{A B}$ as follows:

$$
\begin{array}{r}
S_{A B} \sim \mathcal{N}\left(\bar{S}_{A B}, \Sigma_{A B}\right) \\
\bar{S}_{A B}=\left(\begin{array}{c}
\left(x_{B}+x_{A}\right) / 2 \\
\left(y_{B}+y_{A}\right) / 2
\end{array}\right) \\
\Sigma_{A B}=V L V^{-1} \\
V=\left(\begin{array}{cc}
x_{B}-x_{A} & y_{A}-y_{B} \\
y_{B}-y_{A} & x_{B}-x_{A}
\end{array}\right) \\
L=\left(\begin{array}{cc}
\lambda_{1} & 0 \\
0 & \lambda_{2}
\end{array}\right)=\left(\begin{array}{ll}
\frac{|A B|^{2}}{4} & 0 \\
0 & \frac{e_{s}^{2}}{4}
\end{array}\right)
\end{array}
$$

With $e_{s}$ being the thickness of every link, $\lambda_{1}$ and $\lambda_{2}$ the eigenvalues of the the matrix $V$. Thus the probability we are looking for is defined as follows:

$$
C_{p}=P_{A B}=\int \mathcal{N}\left(\underline{\bar{X}}, \Sigma_{X}\right) \mathcal{N}\left(\bar{S}_{A B}, \Sigma_{A B}\right) d X
$$

The product of two Gaussian distributions is a denormalized Gaussian distribution, we can rewrite (19) as follows:

$$
C_{p}=\int \mathcal{N}\left(\underline{\bar{X}}, \Sigma_{X}\right) \mathcal{N}\left(\bar{S}_{A B}, \Sigma_{A B}\right) d X=\int k \mathcal{N}(\underline{\mu}, \Sigma) d X
$$

Using the canonical representation for a Gaussian [18], we get:

$$
C_{p}=k=\frac{1}{p} \exp \left(g_{1}+g_{2}+\frac{1}{2} \underline{\mu}^{T} \Sigma^{-1} \underline{\mu}\right)
$$

with:

$$
\begin{aligned}
& \Sigma=\left[\Sigma_{X}^{-1}+\Sigma_{A B}^{-1}\right]^{-1} \\
& \underline{\mu}=\left[\Sigma_{X}^{-1}+\Sigma_{A B}^{-1}\right]^{-1}\left[\Sigma_{X}^{-1} \bar{X}+\Sigma_{A B}^{-1} \bar{S}_{A B}\right] \\
& p=\log \left[(2 \pi)^{-n / 2}|\Sigma|^{-1 / 2}\right] \\
& g_{1}=\log \left[(2 \pi)^{n / 2}\left|\Sigma_{X}\right|^{-1 / 2}\right]-\frac{1}{2} \bar{X}^{T} \Sigma_{X}^{-1} \frac{\bar{X}}{1} \bar{S}_{A B}^{T} \Sigma_{A B}^{-1} \bar{S}_{A B} \\
& g_{2}=\log \left[(2 \pi)^{n / 2}\left|\Sigma_{A B}\right|^{-1 / 2}\right]-\frac{1}{2}
\end{aligned}
$$

\section{Navigation history}

The criteria calculated above do not take into account the relationship between two successive position measurements. Another criterion defining this relationship is introduced. This one is based on a weighting concept, if a Way is candidate at two consecutive time measurement intervals $\left(t_{k-1}\right.$ and $\left.t_{k}\right)$, then this Way is more likely to be the selected as the correct one. Figure 7 illustrates this case, the Way $\left(W_{1}\right)$ is more likely to be the correct Way than the Way $\left(W_{3}\right)$ at time $t_{k}$.

We update the previous criteria calculated on Section III-B by adding a weight $w$ as follows:

$$
C n_{i}=C_{i} \times w, \quad i \in[d, m, p]
$$


TABLE II: Results for the accuracy rate on lanes number estimation for every criterion (February 2018)

\begin{tabular}{lccc}
\hline & $C_{d}$ & $C_{m}$ & $C_{p}$ \\
\hline Correct estimations & $79 \%$ & $82.95 \%$ & $81.58 \%$ \\
\hline Wrong estimations & $13.89 \%$ & $11.37 \%$ & $9.72 \%$ \\
\hline Zero estimations & $7.11 \%$ & $5.68 \%$ & $8.70 \%$ \\
\hline
\end{tabular}

Where $w$ is denoted as follows:

$$
w \propto \frac{L}{v}
$$

With $v$ the speed of the vehicle from the GPS receiver and $L$ the length of the Way. Finally when the map matching is completed, the correct Way on which the vehicle travels is selected. As discussed in section II-B every Way has a unique identification number, knowing this identification number allows us to extract the number of lanes (Tag 'lanes') from the OSM geodata. However since the OSM is a collaborative project by volunteers, the Tag 'lanes' is not available in all Ways. With the objective of improving the results obtained on lanes number a heuristic has been made. The number of lanes is stored for a distance of $200 \mathrm{~m}$ when no new information about the lanes number is available.

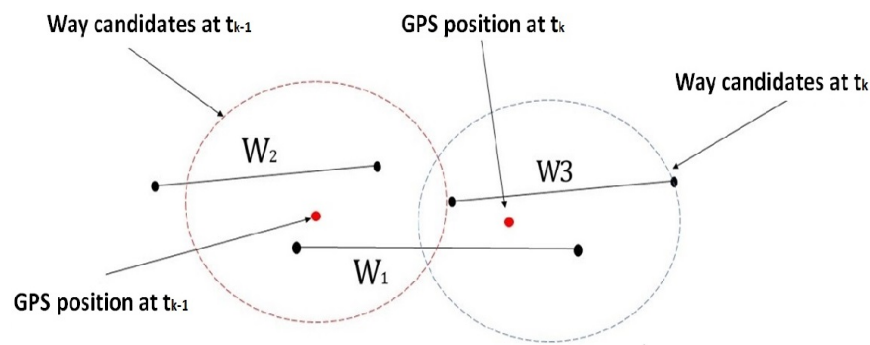

Fig. 7: Example of taking into account the navigation history

\section{REAL-WORLD EXPERIMENTAL RESULTS}

The GPS data were collected in the region of Paris for a total of 6596 GPS points. The GPS receiver provides a decametric accuracy measurement of the longitudinal and lateral coordinates, in addition to the speed of the vehicle and the DOP. The GPS data are received with a frequency of $1 \mathrm{~Hz}$. In order to evaluate the performance of the proposed algorithm, We get the ground truth from the video of a camera installed in front of the vehicle. The results obtained are shown on Figure 8. We divided the incorrect lanes number estimations in two groups. The first one "Zero estimations" denotes the absence of information about the number of lanes from the OSM geodata. In this case the algorithm provides zero for lanes number estimation. The second one "Wrong estimations" indicates the false estimations that are different from "zero estimation" group.

Depending on the criterion used for the map matching, the results for the accuracy rate are shown in the Table II. The map matching based on the Euclidean distance $C_{d}$ provides
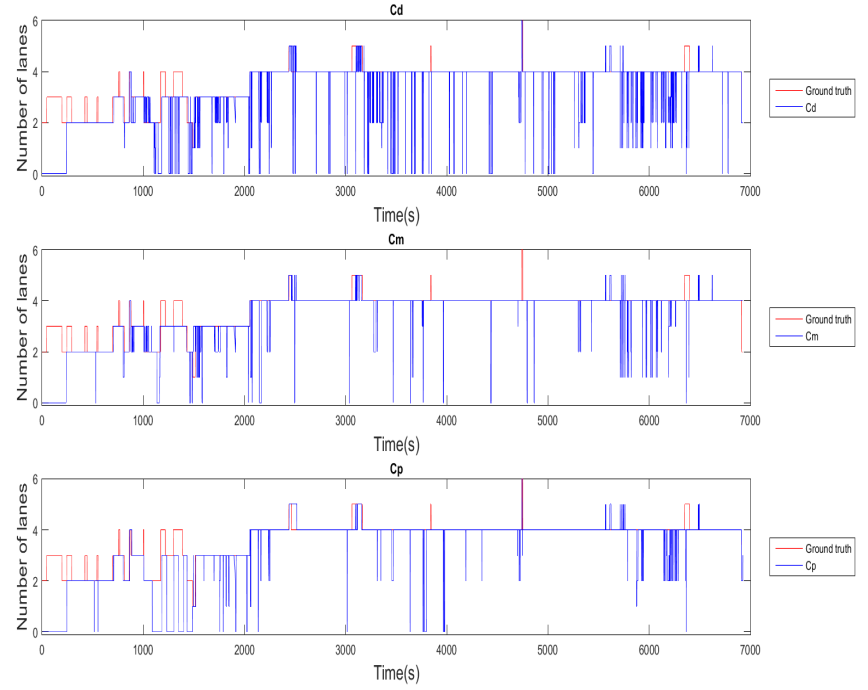

Fig. 8: Lanes estimation compared to ground truth for every criterion used in the map matching.

the less accurate results, which makes sense since this probabilistic criterion uses only inaccurate GPS measurements to calculate the uncertainty about the pose of the vehicle and the orientation of the vehicle. On the other hand the two other criterion $C p$ and $C m$ provide better results considering the use of the vehicle sensors for computing the uncertainty about the pose of the vehicle.

As discussed before the zero estimations are due to the absence of information about the lanes number in the OSM geodata. In order to prove it we run the same algorithm with the same data sets on a different period (April 2018). During the time interval between the two runs, the OSM map has been updated and therefore the map should provide more information about the lanes number. The results obtained on Table III support our argument. As we expected the zero estimations has decreased for each criterion used. In addition the wrong estimations have also decreased, meaning that the wrong estimations are not only due to a wrong map matching. Based on these results, we believe that there are basically three reasons for the lanes number estimation to be false:

- The map matching is correct but the tag "lanes" does not exist for the selected Way (the zero estimation)

- The map matching is correct but the information "number of lanes" is wrong or has not being updated.

- The map matching is false because the wrong Way was selected

For the first and second cases, the results of the lanes number estimation depend on the quality of the OSM map as explained in the previous paragraph. However, it is more difficult to distinguish between the second and the third cases when the number of lanes estimated is wrong. Nevertheless, after investigating on situations where our lanes number estimation is incorrect because of a wrong map matching, we figured out that these cases happen when an exit lane 
TABLE III: Results for the accuracy rate on lanes number estimation for every criterion (April 2018)

\begin{tabular}{lccc}
\hline & $C_{d}$ & $C_{m}$ & $C_{p}$ \\
\hline Correct estimations & $79.85 \%$ & $83.64 \%$ & $83.14 \%$ \\
\hline Wrong estimations & $13.18 \%$ & $11.47 \%$ & $8.51 \%$ \\
\hline Zero estimations & $6.97 \%$ & $4.89 \%$ & $8.35 \%$ \\
\hline
\end{tabular}

occurs as illustrated on Figure 9. In these situations, knowing the future positions of the vehicle would solve the issue. However, in this work, even if the map matching was performed in a post-processing way, we did not use the knowledge about the future positions of the vehicle to correct this false map matching.

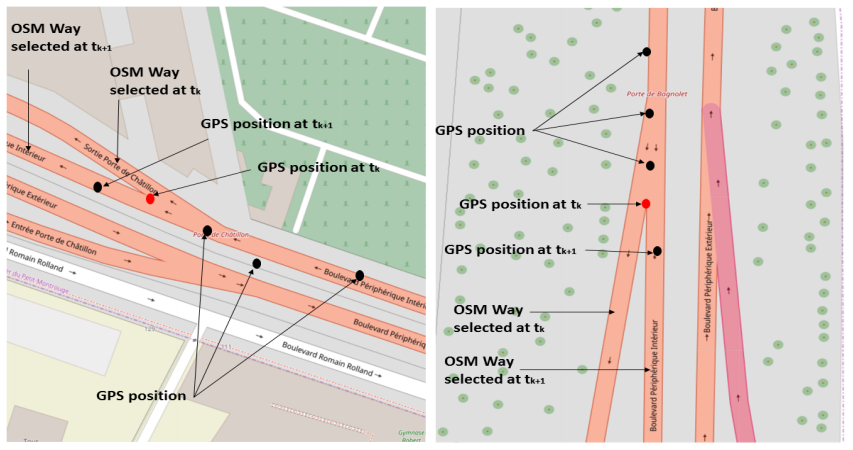

Fig. 9: Scenarios where the map matching algorithm selects the wrong OSM Way and leads to a false lanes number estimation

\section{CONCLUSION}

In this paper we presented a method for estimating the number of lanes in a road using a GPS receiver and the OSM geodata. We match the GPS traces with the existing map according to a probabilistic multicriteria map matching algorithm that takes into account several factors like distance, uncertainties on GPS data, angle between trace and road and the speed limitation. In order to choose the correct road when two roads are close to each other and have a similar direction, the probabilistic criterion is calculated. Three methods for calculating this criterion were proposed depending on the availability of sensors on the vehicle. We also estimated the lanes numbers by extracting the information about the number of lanes from the OSM database. To improve the results, a heuristic about the change in lanes number has been used. In this end, we were able to get up to a $83.64 \%$ correct rate estimation for a set of 6596 GPS points.

We plan to use this map matching algorithm on other maps in order to compare the results. In order to do that, we intend to implement a unique criterion for the map matching, which uses all the three criterion presented in this paper. We also plan to implement our algorithm in real time. Indeed, our algorithm was performed on the integral data set under Matlab for a computation time of 12 minutes and $30 \mathrm{sec}$ on a intel core i7-6820HQ CPU $2.70 \mathrm{GHz}$. Furthermore, we conclude that getting the number of lanes using only OSM is not sufficient. The information about the number of lanes is tributary to selected region and the contribution of volunteers on this region. Therefore, for our future work we want to use the information about the number of lanes as prior for a information driven lanes recognition process using a visionbased sensors [19].

\section{REFERENCES}

[1] HABENICHT, Stefan, WINNER, Hermann, BONE, Sven, et al. A maneuver-based lane change assistance system. In : Intelligent Vehicles Symposium (IV), 2011 IEEE. IEEE, 2011. p. 375-380.

[2] D. Iberraken, L. Adouane, and D. Denis, Multi-level bayesian decision-making for safe and flexible autonomous navigation in highway environment, in 2018 IEEE/RSJ International Conference on Intelligent Robots and Systems (IROS), IEEE, 2018. In press.

[3] KANG, Seokjun et HAN, Dong Seog. Traffic Lane Estimation using Road Width Information. In: Consumer Electronics-Berlin (ICCEBerlin), 2017 IEEE 7th International Conference on.IEEE, 2017.

[4] LIU, Huajun, WANG, Cailing, et YANG, Jingyu. Vanishing points estimation and lanes identification based on Bayesian posterior probability. In : Robotics and Biomimetics (ROBIO), 2014 IEEE International Conference on. IEEE, 2014. p. 867-872.

[5] DAHYOT, Rozenn. Statistical hough transform. IEEE Transactions on pattern analysis and machine intelligence, 2009, vol.31.

[6] LIU, Guoliang, WRGTTER, Florentin, et MARKELI?, Irene. Combining statistical hough transform and particle filter for robust lane detection and tracking. In : Intelligent Vehicles Symposium (IV), 2010 IEEE. IEEE, 2010. p. 993-997.

[7] HARTMANN, Oliver, GABB, Michael, SCHWEIGER, Roland, et al Towards autonomous self-assessment of digital maps. In : Intelligent Vehicles Symposium Proceedings, 2014 IEEE, 2014. p. 89-95.

[8] ZHANG, Lijuan, THIEMANN, Frank, et SESTER, Monika. Integration of GPS traces with road map. In : Proceedings of the Third International Workshop on Computational Transportation Science. ACM, 2010. p. 17-22.

[9] ZHANG, Siqie, LI, Changle, et ZHOU, Xun. A Novel Approach for Mining Road Information from Low Precision GPS Data. In Networking and Network Applications (NaNA), 2017 International Conference on. IEEE, 2017. p. 268-273.

[10] HAKLAY, Mordechai et WEBER, Patrick. Openstreetmap: Usergenerated street maps. IEEE Pervasive Computing, 2008, vol.7 no 4.

[11] HENTSCHEL, Matthias et WAGNER, Bernardo. Autonomous robot navigation based on openstreetmap geodata. In : Intelligent Transportation Systems (ITSC), 2010 13th International IEEE Conference on. IEEE, 2010. p. 1645-1650.

[12] Karim El Mokhtari. Estimation circulaire multi-modèles appliquée au Map matching en environnement contraint. Vision par ordinateur et reconnaissance de formes [cs.CV]. Université du Littoral Côte d?Opale, 2015. Franais. <NNT : 2015DUNK0367>. < tel-01260019>

[13] QUDDUS, Mohammed A. High integrity map matching algorithms for advanced transport telematics applications. 2006. Thèse de doctorat. Imperial College London.

[14] KIM, Wuk, JEE, Gyu-In, et LEE, JangGyu. Efficient use of digital road map in various positioning for ITS. In : Position Location and Navigation Symposium, IEEE 2000. IEEE, 2000. p. 170-176.

[15] FENG, Tao et TIMMERMANS, Harry JP. Map matching of GPS data with Bayesian belief networks. Journal of the Eastern Asia Society for Transportation Studies, 2013, vol. 10, p. 100-112.

[16] RAMM, Frederik, TOPF, Jochen, et CHILTON, Steve. OpenStreetMap: using and enhancing the free map of the world. Cambridge : UIT Cambridge, 2011.

[17] DUDA, Richard O., HART, Peter E., et STORK, David G. Pattern classification. John Wiley \& Sons, 2012

[18] MURPHY, Kevin Patrick et RUSSELL, Stuart. Dynamic bayesian networks: representation, inference and learning. 2002, University of California, Berkeley.

[19] AUFRERE, Romuald, CHAPUIS, Roland, et CHAUSSE, Frédric. A model-driven approach for real-time road recognition. Machine Vision and Applications, 2001, vol. 13, no 2, p. 95-107. 